\title{
Mutational spectrum of CENP-B box and $\alpha$-satellite DNA on chromosome 21 in Down syndrome children
}

\author{
QIAN CHEN ${ }^{1 *}$, BIN TAN $^{2 *}$, JUN-LIN HE $^{3}$, XUE-QING LIU $^{3}$, XUE-MEI CHEN ${ }^{3}$, \\ RU-FEI GAO ${ }^{3}$, JING ZHU ${ }^{2}$, YING-XIONG WANG ${ }^{3 *}$ and HONG-BO QI ${ }^{1 *}$ \\ ${ }^{1}$ Department of Obstetrics and Gynecology, The First Affiliated Hospital of Chongqing Medical University, \\ Chongqing 400016; ${ }^{2}$ Pediatrics Research Institute, Children's Hospital of Chongqing Medical University, \\ Ministry of Education Key Laboratory of Child Development and Disorders, Chongqing 400014; \\ ${ }^{3}$ Laboratory of Reproductive Biology, Public Health College, Chongqing Medical University, Chongqing 400016, P.R. China
}

Received December 16, 2015; Accepted January 13, 2017

DOI: $10.3892 / \mathrm{mmr} .2017 .6247$

\begin{abstract}
The centromere is responsible for the correct inheritance of eukaryotic chromosomes during cell division. Centromere protein B (CENP-B) and its 17 base pair binding site (CENP-B box), which appears at regular intervals in centromeric $\alpha$-satellite DNA ( $\alpha$-satDNA), are important for the assembly of the centromere components. Therefore, it is conceivable that CENP-B box mutations may induce errors in cell division. However, the association between the deoxynucleotide alterations of the CENP-B box and the extra chromosome $21(\mathrm{Chr} 21)$ present in patients with Down syndrome (DS) remains to be elucidated. The mutational spectrum of the $\alpha$-satDNA, including 4 functional CENP-B boxes in Chr21 from 127 DS and 100 healthy children were analyzed by direct sequencing. The de novo occurrences of mutations within CENP-B boxes in patients with DS were excluded. The prevalence of 6 novel mutations (g.661delC, g.1035_1036insA, g.1076_1077insC, g.670T>G, g.1239A>T, g.1343T $>$ C) and 3 single nucleotide polymorphisms (g.727C/T, g.863A/C, g.1264C/G) were not significantly different between
\end{abstract}

Correspondence to: Dr Hong-Bo Qi, Department of Obstetrics and Gynecology, The First Affiliated Hospital of Chongqing Medical University, 1 Youyi Road, Chongqing 400016, P.R. China

E-mail: qihongbo_cqmu@yahoo.com

Professor Ying-Xiong Wang, Laboratory of Reproductive Biology, Public Health College, Chongqing Medical University, 1 Yixueyuan Road, Chongqing 400016, P.R. China

E-mail: wangyingxiong@yahoo.com

*Contributed equally

Abbreviations: $\alpha$-satDNA, $\alpha$-satellite DNA; CENP-B, centromere protein B; Chr21, chromosome 21; DS, Down syndrome; HAC, human artificial chromosome; PCR, polymerase chain reaction

Key words: Down syndrome, mutation, centromere protein B box, $\alpha$-satellite DNA
DS and controls $(\mathrm{P}>0.05)$. However, g.525C/G $(\mathrm{P}=0.01)$, g.601T/C $(\mathrm{P}=0.00000002)$, g.1279A/G $(\mathrm{P}=0.002)$, g.1294C/T $(\mathrm{P}=0.0006)$ and g.1302 $\mathrm{G} / \mathrm{T}(\mathrm{P}=0.004)$ were significantly associated with the prevalence of DS $(\mathrm{P}<0.05)$. The results indicated that CENP-B boxes are highly conserved in DS patients and may not be responsible for $\mathrm{Chr} 21$ nondisjunction events. However, $\alpha$-satDNA in Chr21 is variable and deoxynucleotide deletions, mutations and polymorphisms may act as potential molecular diagnostic markers of DS.

\section{Introduction}

Down syndrome (DS), or trisomy 21 , results from the presence of all or part of an extra chromosome (Chr) 21 and is a high-incidence birth defect that is often associated with physical disorders (1-3) that have no explicit mechanism. Incorrect partitioning of sister chromatids to the daughter cells may induce aneuploidy, including trisomy $21(4,5)$. Centromeres, the evident structures that are located in the center of chromosomes are responsible for chromosome segregation. A previous study indicated that the highly conserved centromere protein B (CENP-B) and its 17 base pair (bp) binding site (CENP-B box) are required for de novo mammalian artificial chromosome formation (6). In vivo analyses with cultured human cells suggested that the presence of the CENP-B box is essential for the formation of functional centromere components $(7,8)$. In addition, CENP-B binding to the CENP-B boxes influences nucleosome positioning in centromeric regions and this binding is required for accurate chromosome segregation (9-11). Therefore, this suggests that nondisjunction of Chr21 may be induced by a defective functioning of the centromere with a CENP-B box mutation.

To determine whether the mutation or absence of the CENP-B box is involved with extra Chr21 formation in DS, the CENP-B boxes in Chr21 $\alpha$-satellite DNA ( $\alpha$-satDNA) of 127 DS children were investigated using polymerase chain reaction (PCR) and direct sequencing. The observations of the present study provide an insight into the molecular association between the CENP-B box and $\alpha$-satDNA in Chr21, which may be a major contributor to the occurrence of trisomy 21 . 


\section{Materials and methods}

Patient recruitment. A total of 127 Chinese children with DS were recruited from the Children's Hospital of Chongqing Medical University (Chongqing, China). Cytogenetic analysis demonstrated the karyotypes were $47, \mathrm{XY},+21$ or $47, \mathrm{XX}$, +21 in all the patients. In addition, 100 healthy children with normal karyotypes were also enrolled as the control group. The distribution of age and residential placement did not differ between controls and DS patients. Peripheral blood samples were collected from all participants. The study was approved by the Ethics Committee of Chongqing Medical University. Informed consent was obtained from all individual participants included in the study.

PCR amplification and sequencing. Genomic DNA was extracted from peripheral blood samples with TIANamp Blood DNA kit (Tiangen Biotech Co., Ltd., Beijing, China). $\alpha$-satDNA (GenBank accession no. D29750.1) in the centromeric region of Chr21 was amplified as two overlapping fragments, with each fragment containing two CENP-B boxes (Fig. 1). Oligonucleotide primer sequences were as follows: $\alpha$-satellite part A, forward 5'-GGAATATCGTCATACAAAAT-3', reverse 5'-TATCAA TGGCAAAGTTCA-3'; and $\alpha$-satellite part B, forward 5'-GTT GAACTTTGCCATTGA-3', and reverse, 5'-GCTCTAAGA AAGCGAATG-3'. PCR was performed using the Takara $\mathrm{Ta}^{\mathrm{TM}}$ PCR kit (Takara Biotechnology, Co., Ltd., Dalian, China), according to the manufacturer's protocol, using $200 \mathrm{ng}$ genomic DNA and $20 \mathrm{nM}$ primers $(1 \mu \mathrm{l})$. PCR thermocycling conditions were as follows: $94^{\circ} \mathrm{C}$ for $4 \mathrm{~min}$ (initial denaturation), followed by 30 cycles at $95^{\circ} \mathrm{C}$ for $30 \mathrm{sec}$ (denaturation), $46^{\circ} \mathrm{C} / 43^{\circ} \mathrm{C}$ for $30 \mathrm{sec}$ (primer annealing) for part $\mathrm{A} / \mathrm{B}$, respectively, and $72^{\circ} \mathrm{C}$ for $45 \mathrm{sec}$ (PCR product elongation), a final extension step was at $72^{\circ} \mathrm{C}$ for $5 \mathrm{~min}$. PCR products were run on $1.5 \%$ agarose gels to detect possible large sequence rearrangements. Following the removal of unincorporated primers and excess dNTPs by exonuclease I and shrimp alkaline phosphatase, the amplified fragments were directly sequenced on an ABI-3100 Genetic Analyzer (Applied Biosystems; Thermo Fisher Scientific, Inc., Waltham, MA, USA). Each sample was compared with NCBI RefSeq sequence of $\alpha$-satDNA.

Statistical analysis. Statistical analysis was used to summarize the clinical characteristics of the subjects. Associations between polymorphisms of DS and control group were examined using the Chi-square test. $\mathrm{P}<0.05$ was considered to indicate a statistically significant difference. All data were analyzed using SPSS software, version 16.0 (SPSS, Inc., Chicago, IL, USA).

\section{Results}

Gel electrophoresis. The gel electrophoresis indicated that the 773 and 535 bp PCR fragments from $\alpha$-satDNA were amplified correctly. There was no observation of a mispairing present in the agarose gels. There was no visible difference in gel electrophoresis between amplified fragments of DS and healthy children (Fig. 2). To examine whether the two fragments exhibited a point mutation, direct sequencing was performed following gel electrophoresis.
A

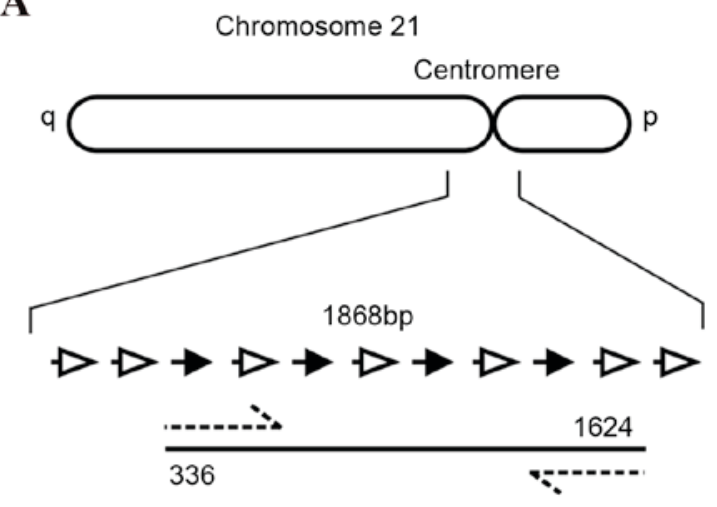

B

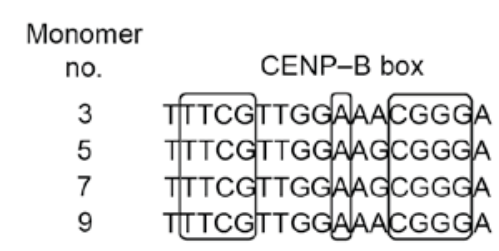

Figure 1. Configuration of the chromosome 21 centromere and the nucleotide sequences of CENP-B boxes. (A) The arrows indicate 11-monomer repeating units; the black arrows indicate $171 \mathrm{bp}$ monomers with a functional CENP-B box. The black bold line with two dash lines refers to sequencing area in $\alpha$-satellite DNA. (B) The 17 bp motif of the CENP-B box. The nucleotide sequences in frames are required for CENP-B binding activity. CENP-B, centromere protein $\mathrm{B}$.
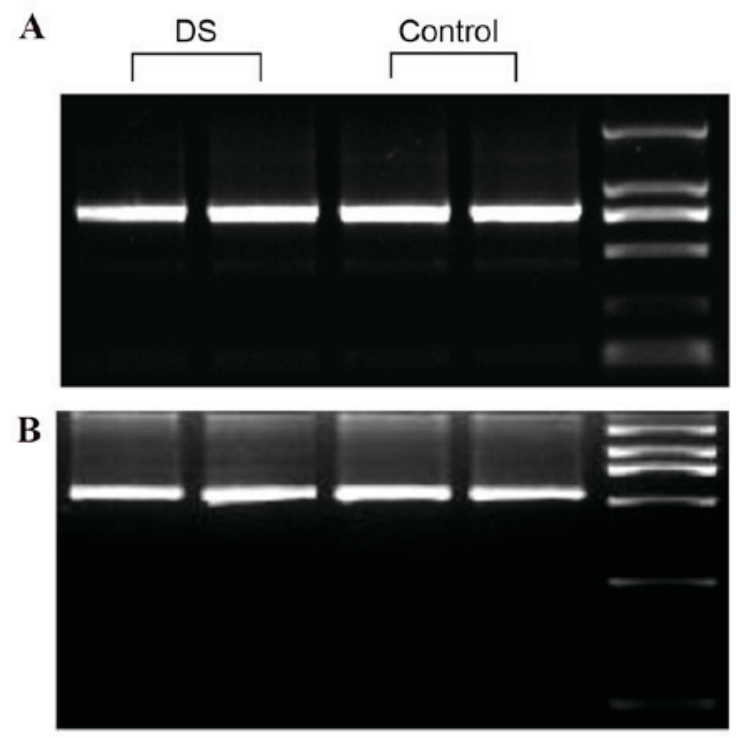

Figure 2. PCR fragments of $\alpha$-satellite DNA in DS and healthy control children. The (A) 773 bp and (B) 535 bp products contain two CENP-B boxes. Marker: DL2000. DS, Down syndrome; PCR, polymerase chain reaction.

CENP-B boxes in Chr21 are highly conserved. The affected patients with DS and healthy children underwent DNA analysis by sequencing of PCR products, of four CENP-B boxes that are respectively located at the g.493_509, g.835_851, g.1173_1189 and g.1511_1527 sites of $\alpha$-satDNA in Chr21. No mutation or absence of CENP-B boxes in the 127 patients with DS and 100 healthy children was observed. All four CENP-B boxes in Chr21 were demonstrated to be highly conserved (Fig. 3). 


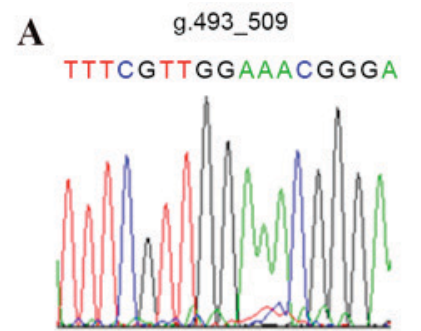

B tTtcgttggaAcGga

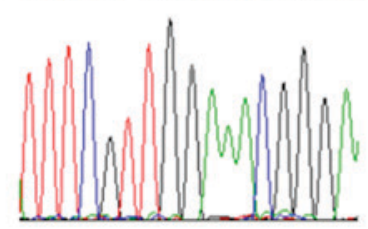

g.835_851

TTTCGTTGGAAGCGGGA

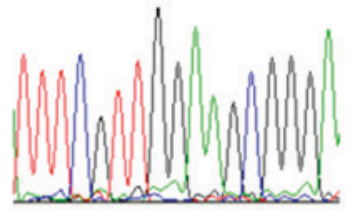

TTTCGTTGGAAGCGGGA

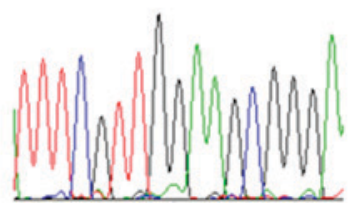

g.1173_1189

TTTCGTTGGAAGCGGGA

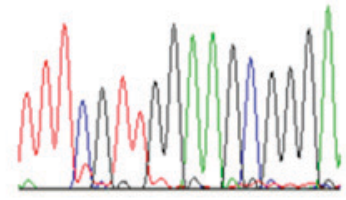

TTTCGTTGGAAGCGGGA

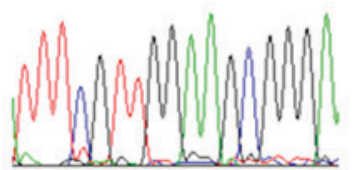

g.1511_1527

TTTCGTTGGAAACGGGA

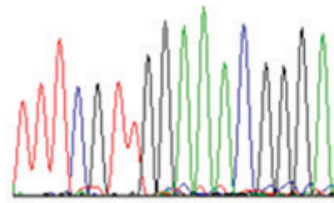

TTTCGTTGGAAACGGGA

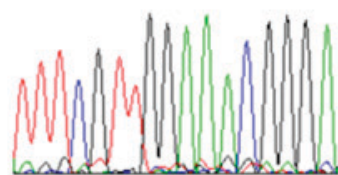

Figure 3. Chromatograms of the four CENP-B boxes. The g.493_509, g.835_851, g.1173_1189 and g.1511_1527 sites of CENP-B boxes in (A) DS patients and (B) healthy children.

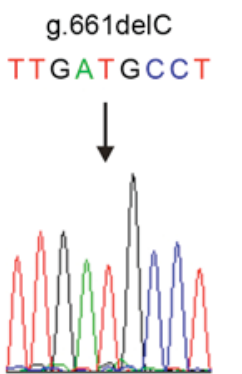

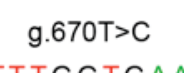
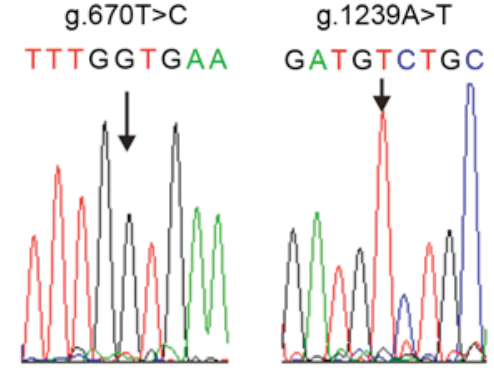
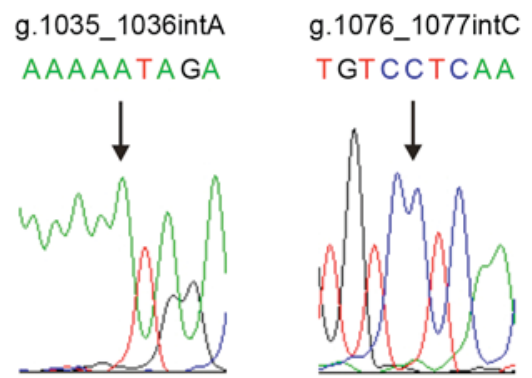

Figure 4. Chromatograms of six mutation sites in $\alpha$-satellite DNA including g.661delC, g.670T>G, g.1239A >T, g.1343T>C, g.1035_1036insA and g.1076_1077insC. Mutation sites are indicated by black arrows.

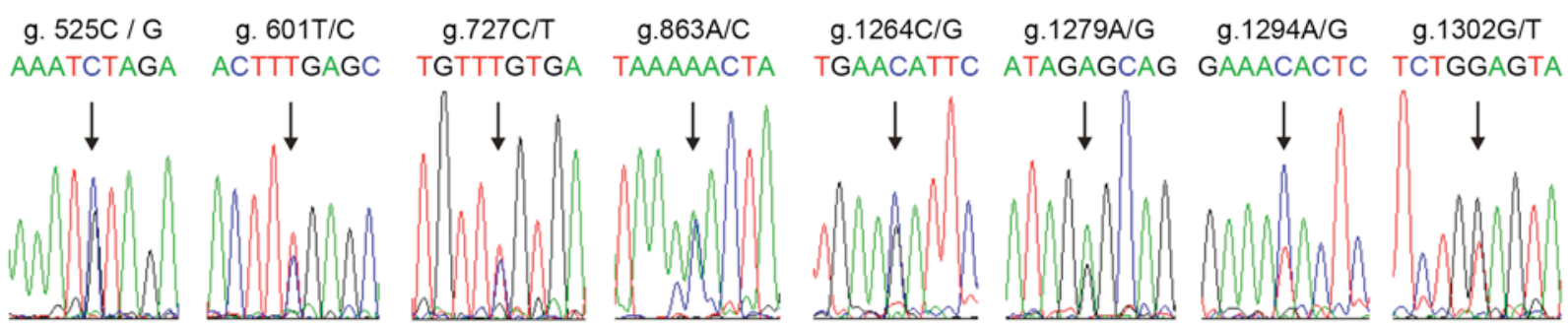

Figure 5. Chromatograms of eight heterozygous sites in $\alpha$-satellite DNA including g.525C/G, g.601T/C, g.727C/T, g.863A/C, g.1264C/G, g.1279A/G, g.1294C/T and g.1302 G/T. Mutation sites are indicated by black arrows.

$\alpha$-satDNA sequence is highly variable. The direct sequencing of $\alpha$-satDNA indicated numerous underlying nucleotide alterations. The variations were point nucleotide deletions, substitutions, insertions and polymorphisms. The novel identified mutations were as follows: g.661delC, g.670T>G, g.1239A>T, g.1343T>C, g.1035_1036insA and g.1076_1077insC (Fig. 4). All the mutations were observed to be present in DS and control groups, however statistical analysis suggested that there was no significant difference between their occurrence in the two groups $(\mathrm{P}>0.05$; Table I). Furthermore, 8 polymorphism sites were observed in the majority of samples and to the best of our knowledge, these had not previously been reported. However, occurrence of the g.525C/G ( $\mathrm{P}=0.01)$, g.601T/C $(\mathrm{P}=0.00000002)$, g.1279A/G
( $\mathrm{P}=0.002)$, g.1294C/T ( $\mathrm{P}=0.0006)$ and g.1302 G/T ( $\mathrm{P}=0.004)$ sites in the DS group demonstrated a significant difference compared with the control group $(\mathrm{P}<0.05)$ However, no differences in g.727C/T $(\mathrm{P}=0.36)$, g.863A/C $(\mathrm{P}=0.207)$ and g.1264C/G $(P=0.073)$ sites were observed between the two groups (Fig. 5; Table I).

\section{Discussion}

DS is a common chromosomal disorder in human aneuploidy, and a termination of pregnancy occurs for the majority DS fetuses identified via Down's screening and amniocentesis. The extra Chr21 results from a meiotic nondisjunction event, with $88 \%$ from nondisjunction in the maternal gamete and 
Table I. Spectrum of $\alpha$-satDNA variants detected in DS and control children.

\begin{tabular}{|c|c|c|c|}
\hline Mutation characteristics & DS $(n=127)$ & Control $(\mathrm{n}=100)$ & P-value \\
\hline g.661delC & $127(100 \%)$ & $100(100 \%)$ & 0.904 \\
\hline g.670T>G & $127(100 \%)$ & $100(100 \%)$ & 0.904 \\
\hline g.1239A $>T$ & $124(97.6 \%)$ & $100(100 \%)$ & 0.122 \\
\hline g. $1343 \mathrm{~T}>\mathrm{C}$ & $124(97.6 \%)$ & $100(100 \%)$ & 0.122 \\
\hline g.1035_1036insA & $125(98.4 \%)$ & $97(97 \%)$ & 0.468 \\
\hline g.1076_1077insC & $125(98.4 \%)$ & $97(97 \%)$ & 0.468 \\
\hline g. $525 \mathrm{C} / \mathrm{G}$ & $125(98.4 \%)$ & $91(91 \%)$ & $0.01^{\mathrm{a}}$ \\
\hline g.601T/C & $93(73.2 \%)$ & $100(100 \%)$ & $0.00000002^{2}$ \\
\hline g. $727 \mathrm{C} / \mathrm{T}$ & $120(94.5 \%)$ & $97(97 \%)$ & 0.36 \\
\hline g. $863 \mathrm{~A} / \mathrm{C}$ & $125(98.4 \%)$ & $100(100 \%)$ & 0.207 \\
\hline g. $1264 \mathrm{C} / \mathrm{G}$ & $123(96.9 \%)$ & $100(100 \%)$ & 0.073 \\
\hline g.1279A/G & $101(79.5 \%)$ & $94(94 \%)$ & $0.002^{\mathrm{a}}$ \\
\hline g. $1294 \mathrm{C} / \mathrm{T}$ & $105(82.7 \%)$ & $97(97 \%)$ & $0.0006^{\mathrm{a}}$ \\
\hline g.1302G/T & $103(81.1 \%)$ & $94(94 \%)$ & $0.004^{\mathrm{a}}$ \\
\hline
\end{tabular}

${ }^{\text {a }}<<0.05$ vs. control. $\alpha$-satDNA, $\alpha$-satellite DNA; DS, Down syndrome.

$8 \%$ in the paternal gamete. But the underlying molecular genetics mechanism linking nondisjunction to $\mathrm{Chr} 21$ remains to be fully elucidated. Therefore, it is necessary to determine the genotypes of DS to provide a methodology for antenatal examination and gene diagnosis for trisomy 21 in noninvasive prenatal testing.

The current study identified that the CENP-B boxes are highly conserved in DS and healthy children. Human Chr21 contains a large repetitive sequence and consists of 11 tandem repeats of an AT-rich 171 bp alphoid monomer unit. This $11 \mathrm{mer}$ repeat construct is a $1.3 \mathrm{Mbp}$ higher order $\alpha$-satDNA repeat on the centromeric area. Each monomer has a unique 17 bp sequence, however only five have the CENP-B binding activity known as the CENP-B box $(12,13)$. In the human artificial chromosome (HAC), CENP-B box mutations induced no HAC formation and diminution of CENP-A assembly $(6,11,14)$. CpG methylation and point mutation of the CENP-B box reduced CENP-B binding activity (15-17). Therefore, the CENP-B box is required for de novo centromere chromatin assembly on human $\alpha$-satDNA. The present study identified the methylation status of $\mathrm{CpG}$ dinucleotides in CG islands; however, the results did not show any difference in the DNA methylation status between DS and healthy people as previously described (18).

A cohort of 127 patients diagnosed with DS and 100 healthy children in Chongqing were investigated via chromosome screening. The spectrum of $\alpha$-satDNA variants in Chr21 was analyzed by direct DNA sequencing. The present study identified 14 novel variations that, to the best of our knowledge, have not previously been reported. A total of 6 mutations were identified in the Chinese population: g.661delC, g.670T>G, g.1239A $>$ T, g.1343T >C, g.1035_1036insA and g.1076_1077insC. The present study revealed the distribution and frequency of novel variations among the population in Chongqing. For human Chr21, $\alpha$-satDNA appears to be the major constituent of functional centromeres (19), as it is associated with the existence of functional centromere components on the endogenous human chromosome and the ability to induce de novo centromere assembly. In the present study, the CENP-B box and $\alpha$-satDNA sequence were required for de novo mammalian artificial chromosome formation and assembly of functional centromere components, including CENP-A, CENP-C, and CENP-E $(20,21)$. These results demonstrated the presence of a variable in $\alpha$-satDNA, other than the CENP-B box, which may be important for de novo centromere formation function $(8,16,22,23)$. In addition, the present study reported eight single nucleotide polymorphisms (SNPs) of $\alpha$-satDNA in Chr21. Three SNPs, including g.727C/T, g.863A/C and g.1264C/G did not demonstrate any difference in occurrence between DS and healthy children. It was therefore hypothesized that the variants identified in the present study may be due to ethnic disparities, as suggested by previous studies $(18,24)$ and consequently, these variants may act as DNA markers for distinguishing ethnicities. However, five SNPs including g.525C/G, g.601T/C, g.1279A/G, g.1294C/T and g.1302 G/T were observed to be significantly different between the DS and control group, and may therefore act as potential molecular diagnostic markers of DS.

The present study exhibited various limitations. Firstly, the study was conducted on a small sample size and larger sample sizes are required to confirm these associations in the future. Furthermore, the patients were from the same region of China, therefore further studies should include individuals from different regions of China and of different ethnicities.

In conclusion, the present study investigated the $\alpha$-satDNA variants present in Chr21 and any differences in genotype between DS and healthy children. The results demonstrated that CENP-B box mutations were not present and the box appears to be highly conserved in DS patients and therefore may not be responsible for Chr21 nondisjunction events. Furthermore, 14 novel variations were identified in this investigation and the differing mutation spectrum of $\alpha$-satDNA between DS and 
healthy individuals may contribute to complex DS phenotypes and act as potential dinucleotide markers for diagnosing trisomy 21.

\section{Acknowledgements}

The authors would like to thank Dr Lin Zou and Dr Cui Song (Center of Clinical Molecular Medicine, Children's Hospital of Chongqing Medical University; Chongqing, China) for case collection. The present study was supported by the National Natural Science Foundation of China (grant no. 30771202).

\section{References}

1. Shott SR, Joseph A and Heithaus D: Hearing loss in children with Down syndrome. Int J Pediatr Otorhinolaryngol 61: 199-205, 2001.

2. Nespoli L, Burgio GR, Ugazio AG and Maccario R: Immunological features of Down's syndrome: A review. J Intellect Disabil Res 37: 543-551, 1993.

3. Karlsson B, Gustafsson J, Hedov G, Ivarsson SA and Annerén G: Thyroid dysfunction in Down's syndrome: Relation to age and thyroid autoimmunity. Arch Dis Child 79: 242-245, 1998.

4. Wittmann T, Hyman A and Desai A: The spindle: A dynamic assembly of microtubules and motors. Nat Cell Biol 3: E28-E34, 2001.

5. Walczak CE and Heald R: Mechanisms of mitotic spindle assembly and function. Int Rev Cytol 265: 111-158, 2008.

6. Ohzeki J, Nakano M, Okada T and Masumoto H: CENP-B box is required for de novo centromere chromatin assembly on human alphoid DNA. J Cell Biol 159: 765-775, 2002.

7. Trowell HE, Nagy A, Vissel B and Choo KH: Long-range analyses of the centromeric regions of human chromosomes 13 14 and 21: Identification of a narrow domain containing two key centromeric DNA elements. Hum Mol Genet 2: 1639-1649, 1993.

8. Okada T, Ohzeki J, Nakano M, Yoda K, Brinkley WR, Larionov V and Masumoto H: CENP-B controls centromere formation depending on the chromatin context. Cell 131: 1287-1300, 2007.

9. Tanaka Y, Tachiwana H, Yoda K, Masumoto H, Okazaki T, Kurumizaka $\mathrm{H}$ and Yokoyama $\mathrm{S}$ : Human centromere protein $\mathrm{B}$ induces translational positioning of nucleosomes on alpha-satellite sequences. J Biol Chem 280: 41609-41618, 2005.

10. Hasson D, Panchenko T, Salimian KJ, Salman MU, Sekulic N, Alonso A, Warburton PE and Black BE: The octamer is the major form of CENP-A nucleosomes at human centromeres. Nat Struct Mol Biol 20: 687-695, 2013.
11. Okamoto Y, Nakano M, Ohzeki J, Larionov V and Masumoto H: A minimal CENP-A core is required for nucleation and maintenance of a functional human centromere. EMBO J 26: 1279-1291, 2007.

12. Ikeno M, Masumoto H and Okazaki T: Distribution of CENP-B boxes reflected in CREST centromere antigenic sites on long-range alpha-satellite DNA arrays of human chromosome 21. Hum Mol Genet 3: 1245-1257, 1994.

13. Alkan C, Ventura M, Archidiacono N, Rocchi M, Sahinalp SC and Eichler EE: Organization and evolution of primate centromeric DNA from whole-genome shotgun sequence data. PLoS Comput Biol 3: 1807-1820, 2007.

14. Basu J, Stromberg G, Compitello G, Willard HF and Van Bokkelen G: Rapid creation of BAC-based human artificial chromosome vectors by transposition with synthetic alpha-satellite arrays. Nucleic Acids Res 33: 587-596, 2005.

15. Earnshaw WC, Sullivan KF, Machlin PS, Cooke CA, Kaiser DA, Pollard TD, Rothfield NF and Cleveland DW: Molecular cloning of cDNA for CENP-B, the major human centromere autoantigen. J Cell Biol 104: 817-829, 1987.

16. Masumoto H, Masukata H, Muro Y, Nozaki N and Okazaki T: A human centromere antigen (CENP-B) interacts with a short specific sequence in alphoid DNA, a human centromeric satellite. J Cell Biol 109: 1963-1973, 1989.

17. Tanaka Y, Kurumizaka $\mathrm{H}$ and Yokoyama S: CpG methylation of the CENP-B box reduces human CENP-B binding. FEBS J 272 282-289, 2005

18. Xia YY, Ding YB, Liu XQ, Chen XM, Cheng SQ, Li LB, Ma MF, $\mathrm{He} \mathrm{JL}$ and Wang YX: Allelic methylation status of $\mathrm{CpG}$ islands on chromosome 21q in patients with Trisomy 21. Mol Med Rep 9: $1681-1688,2014$.

19. Ugarković D and Plohl M: Variation in satellite DNA profile-causes and effects. EMBO J 21: 5955-5959, 2002.

20. Sullivan LL, Boivin CD, Mravinac B, Song IY and Sullivan BA: Genomic size of CENP-A domain is proportional to total alpha satellite array size at human centromeres and expands in cancer cells. Chromosome Res 19: 457-470, 2011.

21. Irvine DV, Amor DJ, Perry J, Sirvent N, Pedeutour F, Choo KH and Saffery R: Chromosome size and origin as determinants of the level of CENP-A incorporation into human centromeres. Chromosome Res 12: 805-815, 2004.

22. Meštrović N, Pavlek M, Car A, Castagnone-Sereno P, Abad P and Plohl M: Conserved DNA motifs, including the CENP-B box-like, are possible promoters of satellite DNA array rearrangements in nematodes. PLoS One 8: e67328, 2013.

23. Fachinetti D, Folco HD, Nechemia-Arbely Y, Valente LP, Nguyen K, Wong AJ, Zhu Q, Holland AJ, Desai A, Jansen LE and Cleveland DW: A two-step mechanism for epigenetic specification of centromere identity and function. Nat Cell Biol 15: 1056-1066, 2013

24. Xia YY, Ding YB, Liu XQ, Chen XM, Cheng SQ, Li LB, Ma MF, He JL and Wang YX: Racial/ethnic disparities in human DNA methylation. Biochim Biophys Acta 1846: 258-262, 2014. 